\title{
Combined effects of social relationships and healthful dietary behaviours in adults aged 50 and over
}

\author{
A. Conklin ${ }^{1,2}$, N. Wareham ${ }^{1,2}$, N. Forouhi ${ }^{1}$ and P. Monsivais ${ }^{2}$ \\ ${ }^{1}$ MRC Epidemiology Unit, Cambridge, CB2 OQQ, UK and ${ }^{2}$ Centre for Diet and Activity Research, \\ University of Cambridge, CB2 OSR, UK
}

Social relationships may offer protection against chronic conditions and may influence likelihood of survival. ${ }^{(1)}$ They have also been linked to dietary behaviour which may be an important pathway through which social circumstances impact health. ${ }^{(2)}$ But less is known about structural social ties and healthful dietary behaviours among women and men and to what extent different types of social ties interact to produce a combined influence.

Prospective data from adults ( $\geq 50$ years) in the European Prospective Investigation of Cancer-Norfolk study was used to examine sexspecific associations between structural social ties (marital status, living arrangement, friend contact, family contact) and a dietary score for variety of fruits (ranging between 0-11) or vegetables (0-26) with linear regression. Separate interaction models tested whether overall effects of marital status changed by living arrangement or by friend contact, and of living arrangement by friend contact. Analyses adjusted for energy intake, age, education and, where appropriate, sex.

The negative association of widowhood with vegetable variety was stronger for men (women: Beta $=-0.79, p<0.001 ;$ men: Beta $=-2.17, p<0.001$ ), and similarly regarding lone-living and vegetable variety (women: Beta $=-0.66, p<0.001$; men: Beta $=-1.46$, $p<0.001$ ). Interaction models including both genders revealed the most striking results. Living arrangement modified the association of marital status categories with variety scores, although living arrangement differences across non-partnered categories were not significant. Similarly, compared with being partnered, lower variety scores associated with non-partnered categories were significantly larger when friend contact was infrequent than when frequent (fruit variety: $p=0.034$; vegetable variety: $p=0.026$ ) (Fig. 1). There was also a significant difference $(p=0.007)$ between infrequent friend contact $($ Beta $=-1.62 ; P<0.001)$ and frequent contact $($ Beta $=-0.80$; $P<0.001)$ in lower vegetable variety scores of lone-living adults compared to those living with someone else (Fig. 2).

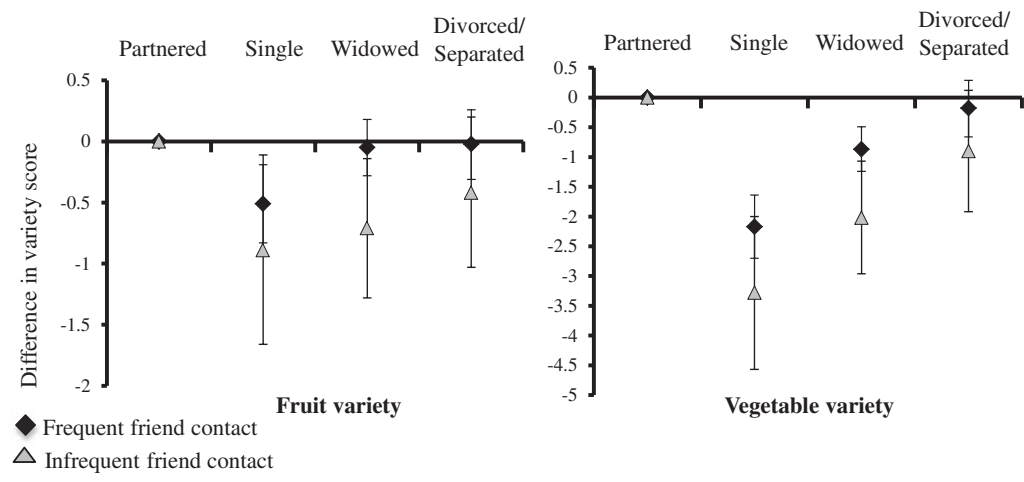

Fig. 1. Contact modifies marital status.

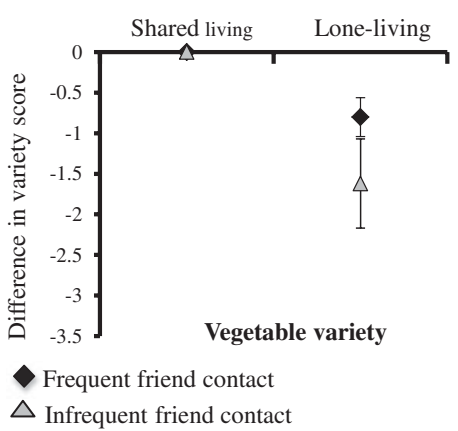

Fig. 2. Contact modifies living arrangement.

Marital status, living arrangement or friend contact influence variety scores more for men than women. Different social ties interact to produce unique synergistic effects on healthful dietary behaviours. Results highlight the importance of considering living arrangement and frequency of social contact when assessing whether widowed or single older adults are at risk of lower fruit and vegetable variety.

1. Holt-Lunstad J, Smith TB \& Layton JB (2010) PLoS Med 7, e1000316.

2. Berkman LF, Glass T, Brissette I, Seeman TE (2000) Social Science \& Medicine 51, 843-57. 